\title{
Fluctuations of central measures on partitions (extended abstract)
}

\author{
Pierre-Loïc Méliot \\ Universität Zürich - Institut für Mathematik - Winterthurerstrasse 190, CH-8057 Zürich - Switzerland \\ pierre-loic.meliot@math.uzh.ch
}

\begin{abstract}
We study the fluctuations of models of random partitions $\left(\mathbb{P}_{n, \omega}\right)_{n \in \mathbb{N}}$ stemming from the representation theory of the infinite symmetric group. Using the theory of polynomial functions on Young diagrams, we establish a central limit theorem for the values of the irreducible characters $\chi^{\lambda}$ of the symmetric groups, with $\lambda$ taken randomly according to the laws $\mathbb{P}_{n, \omega}$. This implies a central limit theorem for the rows and columns of the random partitions, and these "geometric" fluctuations of our models can be recovered by relating central measures on partitions, generalized riffle shuffles, and Brownian motions conditioned to stay in a Weyl chamber.
\end{abstract}

Résumé. Nous étudions les fluctuations de modèles de partitions aléatoires $\left(\mathbb{P}_{n, \omega}\right)_{n \in \mathbb{N}}$ issus de la théorie des représentations du groupe symétrique infini. En utilisant la théorie des fonctions polynomiales sur les diagrammes de Young, nous établissons un théorème central limite pour les valeurs des caractères irréductibles $\chi^{\lambda}$ des groupes symétriques, avec $\lambda$ pris aléatoirement suivant les lois $\mathbb{P}_{n, \omega}$. Ceci implique un théorème central limite pour les lignes et les colonnes des partitions aléatoires, et ces fluctuations "géométriques" de nos modèles peuvent être retrouvées en reliant les mesures centrales sur les partitions, les battages généralisés de cartes, et les mouvements browniens conditionnés à rester dans une chambre de Weyl.

Keywords: Random partitions, generalized riffle shuffles, representation theory of the infinite symmetric group.

Let $G$ be a group, and $\tau$ be a normalized non-negative trace on $G$, that is to say that $\tau$ is a function from $G$ to $\mathbb{C}$ such that $\tau\left(e_{G}\right)=1, \tau(g h)=\tau(h g)$ for any $g$ and $h$, and $\left(\tau\left(g_{i} g_{j}^{-1}\right)\right)_{1 \leq i, j \leq n}$ is Hermitian non-negative definite for any family $\left(g_{1}, \ldots, g_{n}\right)$ in $G$. If $G$ is finite, then $\tau$ can be written uniquely as a non-negative linear combination of the normalized irreducible characters of $G$ :

$$
\tau=\sum_{\lambda \in \widehat{G}} \mathbb{P}_{\tau}[\lambda] \chi^{\lambda}
$$

where $\widehat{G}$ is the set of classes of isomorphism of irreducible complex linear representations of $G, \chi^{\lambda}$ is the normalized character of a representation of class $\lambda$, and the weights $\mathbb{P}_{\tau}[\lambda]$ are non-negative and sum to 1 . The function $\mathbb{P}_{\tau}: \widehat{G} \rightarrow \mathbb{R}_{+}$is called the spectral measure of $\tau$; it is a probability measure on $\widehat{G}$. In the case of the finite symmetric groups $\mathfrak{S}_{n}$, an interesting family of spectral measures can be obtained by restriction of a trace of the infinite symmetric group $\mathfrak{S}_{\infty}=\bigcup_{n=0}^{\infty} \uparrow \mathfrak{S}_{n}$. Recall that the irreducible representations of $\mathfrak{S}_{n}$ are labelled by the set $\mathfrak{P}_{n}$ of integer partitions $\lambda=\left(\lambda_{1} \geq \cdots \geq \lambda_{r}\right)$ of size $n$. On 
the other hand, the set of normalized traces on $\mathfrak{S}_{\infty}$ is a compact convex set, and its extremal points have been classified by Thoma (see [Tho64]). They are labelled by the infinite dimensional Thoma simplex

$$
\Omega=\left\{\omega=\left(\left(\alpha_{i}\right)_{i \geq 1},\left(\beta_{i}\right)_{i \geq 1}\right) \mid \sum_{i \geq 1}\left(\alpha_{i}+\beta_{i}\right)=1-\gamma \leq 1\right\}
$$

where $\alpha$ and $\beta$ are non-increasing sequences of non-negative real numbers. Moreover, the value of the extremal trace $\chi^{\omega}$ of parameter $\omega$ on a permutation $\sigma_{\mu}$ product of disjoint cycles of lengths $\mu_{1}, \ldots, \mu_{r}$ is

$$
\chi^{\omega}\left(\sigma_{\mu}\right)=p_{\mu}(\omega)=p_{\mu_{1}}(\omega) p_{\mu_{2}}(\omega) \cdots p_{\mu_{r}}(\omega),
$$

where the $p_{k}(\omega)$ 's are defined by the specialization of the algebra of symmetric functions $p_{1}(\omega)=1$ and $p_{k \geq 2}(\omega)=\sum_{i=1}^{\infty} \alpha_{i}^{k}+(-1)^{k-1} \beta_{i}^{k}$. We call central measures the spectral measures $\mathbb{P}_{n, \omega}=\mathbb{P}_{\left.\chi^{\omega}\right|_{\mathfrak{S}_{n}}}$ associated to the restrictions of the extremal characters $\chi^{\omega}$ to the finite symmetric groups $\mathfrak{S}_{n}$. Thus,

$$
\chi^{\omega}(\sigma)=\sum_{\lambda \in \mathfrak{P}_{n}} \mathbb{P}_{n, \omega}[\lambda] \chi^{\lambda}(\sigma) \quad \text { for } \sigma \in \mathfrak{S}_{n} .
$$

The central measures are characterized by a property of harmonicity on the Young graph $\mathfrak{P}=\bigsqcup_{n \in \mathbb{N}} \mathfrak{P}_{n}$, and using Frobenius' formula and Thoma's theorem for the values of the extremal characters, one obtains the following abstract formula for the central measures:

$$
\mathbb{P}_{n, \omega}[\lambda]=(\operatorname{dim} \lambda) s_{\lambda}(\omega),
$$

where $\operatorname{dim} \lambda$ is the complex dimension of the Specht module of $\mathfrak{S}_{n}$ of label $\lambda$ (given for instance by the hook-length formula), and $s_{\lambda}(\omega)$ is the specialization of the Schur function $s_{\lambda}$.

The first-order asymptotics of the central measures have been determined by Kerov and Vershik in [KV81]. They have shown that if a partition $\lambda$ is chosen according to a central measure $\mathbb{P}_{n, \omega}$, then

1. for any permutation $\sigma \in \mathfrak{S}_{\infty}, \lim _{n \rightarrow \infty} \chi^{\lambda}(\sigma)=\chi^{\omega}(\sigma)$.

2. the asymptotic frequences of the rows and columns of $\lambda$ are given by the parameters $\alpha_{i}$ and $\beta_{j}$ :

$$
\lambda_{i} / n \rightarrow \alpha_{i} \quad \text { and } \quad \lambda_{j}^{\prime} / n \rightarrow \beta_{j} \quad \text { for } i, j \geq 1 .
$$

Here, the convergence holds in probability, and $\lambda^{\prime}$ denotes the conjugate partition of $\lambda$. Thus, the central measures are "concentrated" around their mean value. The purpose of our article is to show that this concentration is Gaussian; hence, we shall prove that the fluctuations

$$
\sqrt{n}\left(\chi^{\lambda}(\sigma)-\chi^{\omega}(\sigma)\right) \quad ; \quad \sqrt{n}\left(\lambda_{i} / n-\alpha_{i}\right) \quad ; \quad \sqrt{n}\left(\lambda_{j}^{\prime} / n-\beta_{j}\right)
$$

all converge towards centered gaussian processes with explicit covariances. A way to do this is to use the algebra of observables (or polynomial functions) of Young diagrams, in the sense of Kerov and Olshanski. Hence, in Section 11, we will sketch a proof of the gaussian behaviour of the character values that relies on the combinatorics of the Ivanov-Kerov algebra $\mathscr{O}$ (see [IK99]); see Theorem 11. This result implies that the rows and the columns also have an asymptotic gaussian behaviour, but only when the two sequences 
of parameters $\alpha$ and $\beta$ are strictly decreasing (see Theorem 2 , using different methods, Bufetov obtained exactly the same result in [Buf11]).

Unfortunately, the method of polynomial functions cannot be used in the case of equal parameters (for instance when $\alpha_{1}=\alpha_{2}=\cdots=\alpha_{m}=1 / m$ ). This problem and its solution are related to the following question: can one explain the asymptotic gaussian behaviour of the central measures by exhibiting independent random variables to which the usual central limit theorem applies, and that drive the (asymptotic) behaviour of the rows and columns of the random partitions? Sections 2 and 3 of our paper provide an affirmative answer: the independent random variables to be considered will determine choices of decks of cards in generalized riffle shuffles (in the sense of [Ful02]), and the map that allows to recover the random partitions is the Robinson-Schensted-Knuth algorithm. Hence, in Section 2, we shall prove by using the combinatorial algebra FQSym of free quasisymmetric functions that the central measures are the images by the RSK map of measures on the sets of permutations $\mathfrak{S}_{n}$ coming from generalized riffle shuffles (this result has already appeared in the work of Fulman, but our proof is new). In Section 3 , we then use a result of O'Connell ([O'C03]) to relate the RSK algorithm on random words to a transformation of (random) paths called Pitman's transform. This leads to the following correction of Theorem 2 . when some parameters $\alpha_{i}$ or $\beta_{j}$ are equal, the fluctuations of the corresponding rows and columns have a gaussian component and an "interaction component" that is distributed like the eigenvalues of a traceless gaussian Hermitian matrix (see Theorem 4 for a precise statement). Unfortunately, we are for this moment only able to prove the correction when $\gamma=0$ and $\beta$ has a finite number of non-zero parameters; but we hope that our method can be extended to the general case. In Section 4 , we conclude by stating a conjecture that would improve the results of the first paragraph, and provide new estimates at least for the random character values. Hence, it is suspected that these values converge not only after scaling by $n^{1 / 2}$ to gaussian variables, but in fact after scaling by $n^{2 / 3}$ in the (strong) mod-gaussian sense (cf. [JKN11]).

\section{An algebraic central limit theorem for random characters}

For two partitions $\lambda$ and $\mu$ of sizes $n$ and $k$, one introduces the renormalized character value

$$
\Sigma_{\mu}(\lambda)= \begin{cases}n(n-1)(n-2) \cdots(n-k+1) \chi^{\lambda}\left(\sigma_{\mu}\right) & \text { if } n \geq k \\ 0 & \text { otherwise }\end{cases}
$$

where $\sigma_{\mu}$ denotes as before a permutation product of disjoint cycles of lengths $\mu_{1}, \ldots, \mu_{r}$ (see [IO02, Śni06]). The interest of the renormalization factor $n^{\downarrow k}=n(n-1) \cdots(n-k+1)$ is that the symbols $\Sigma_{\mu}$ with $\mu$ in $\mathfrak{P}=\bigsqcup_{n \in \mathbb{N}} \mathfrak{P}_{n}$ generate linearly a algebra of functions on Young diagrams, which is denoted $\mathscr{O}$ and is graded by $\operatorname{deg} \Sigma_{\mu}=|\mu|=\mu_{1}+\cdots+\mu_{r}$. In particular, a product of two functions $\Sigma_{\mu} \Sigma_{\nu}$ is a linear combination $\sum_{\rho} c_{\rho}^{\mu \nu} \Sigma_{\rho}$ of symbols $\Sigma_{\rho}$ with $|\rho| \leq|\mu|+|\nu|$. A combinatorial proof of this fact can be given by interpreting the elements $\Sigma_{\mu}$ as elements of a subalgebra of the combinatorial algebra of partial permutations ([IK99]). Recall that a partial permutation is a pair $(\sigma, S)$ with $S$ finite subset of $\mathbb{N}^{*}$ and $\sigma$ permutation of $S$. The product of two partial permutations is $(\sigma, S)(\tau, T)=(\sigma \circ \tau, S \cup T)$, and one can consider the algebra $\mathscr{P}$ of formal linear combinations of partial permutations, graded by $\operatorname{deg}(\sigma, S)=\operatorname{card} S$. Then, one can identify $\Sigma_{\mu}$ with the formal linear combination

$$
\sum_{a_{11} \neq a_{12} \neq \cdots \neq a_{r \mu_{r}}}\left(\left(a_{11}, \ldots, a_{1 \mu_{1}}\right)\left(a_{21}, \ldots, a_{2 \mu_{2}}\right) \cdots\left(a_{r 1}, \ldots, a_{r \mu_{r}}\right),\left\{a_{i j}\right\}_{(i, j) \in I(\mu)}\right),
$$


where the sum is taken over injective functions from $I(\mu)=\left\{(i, j) \mid 1 \leq i \leq \ell(\mu), 1 \leq j \leq \mu_{i}\right\}$ to $\mathbb{N}^{*}$ : the relations between the functions on Young diagrams $\Sigma_{\mu}$ are the same as the relations ${ }^{(i)}$ between the linear combinations of partial permutations $\Sigma_{\mu}$. This identification yields an explicit description of the product of two symbols $\Sigma$, see [FM10, §3.4]:

$$
\Sigma_{\mu} \Sigma_{\nu}=\sum \Sigma_{\rho(p ; \mu, \nu)}
$$

where the sum is taken over partial matchings $p: I(\mu) \rightarrow I(\nu)$ (one-to-one functions from a subset to a subset), and $\rho(p ; \mu, \nu)$ denotes the cycle type of a product of partial permutations of cycle types $\mu$ and $\nu$ and supports that intersect according to the partial matching. For instance, $\Sigma_{2} \Sigma_{2}$ is a sum over the seven partial matchings between two sets of size 2 , and is equal to $\Sigma_{2,2}+4 \Sigma_{3}+2 \Sigma_{1,1}$.

Now, let us detail the uses of the symbols $\Sigma_{\mu}$ in the asymptotic analysis of the central measures. The expectation of the random variable $\Sigma_{\mu}(\lambda)$ with $\lambda$ chosen randomly according to $\mathbb{P}_{n, \omega}$ is easy to compute:

$$
\mathbb{E}_{n, \omega}\left[\Sigma_{\mu}\right]=\sum_{\lambda \in \mathfrak{P}_{n}} \mathbb{P}_{n, \omega}[\lambda] n^{\downarrow|\mu|} \chi^{\lambda}\left(\sigma_{\mu}\right)=n^{\downarrow|\mu|} \chi^{\omega}\left(\sigma_{\mu}\right)=n^{\downarrow|\mu|} p_{\mu}(\omega) \simeq_{n \rightarrow \infty} n^{|\mu|} p_{\mu}(\omega) .
$$

Because of the explicit product rule (1), it is then natural to compute the (asymptotics of the) moments $\mathbb{E}_{n, \omega}\left[\left(\Sigma_{\mu}\right)^{p}\right]$, and thus to use a method of moments to prove the convergence of

$$
X_{n, \omega}\left(\sigma_{\mu}\right)=\sqrt{n}\left(\chi^{\lambda}\left(\sigma_{\mu}\right)-\chi^{\omega}\left(\sigma_{\mu}\right)\right) \simeq \sqrt{n}\left(\frac{\Sigma_{\mu}(\lambda)}{n^{|\mu|}}-p_{\mu}(\omega)\right)
$$

towards a gaussian variable. Actually, it is better to look at the joint cumulants of the observables of random Young diagrams, defined by

$$
k_{n, \omega}^{(r)}\left(X_{1}, \ldots, X_{r}\right)=\sum_{\llbracket 1, r \rrbracket=\pi_{1} \sqcup \cdots \sqcup \pi_{s} \text { set partition }}(-1)^{s-1}(s-1) ! \prod_{\pi_{j} \in \pi} \mathbb{E}_{n, \omega}\left[\prod_{i \in \pi_{j}} X_{i}\right] .
$$

Indeed, random variables having moments of all order form a gaussian vector if and only if all their joints cumulants $k^{(r)}$ with $r \geq 3$ are equal to zero; and in this case, the covariance matrix of the gaussian vector is given by the second cumulants $k^{(2)}(X, Y)=\mathbb{E}[X Y]-\mathbb{E}[X] \mathbb{E}[Y]=\operatorname{cov}(X, Y)$. However, one can show that for any observables $X_{1}, \ldots, X_{r}$ in $\mathscr{O}$,

$$
k_{n, \omega}^{(r)}\left(X_{1}, \ldots, X_{r}\right)=O\left(n^{\operatorname{deg} X_{1}+\cdots+\operatorname{deg} X_{r}-(r-1)}\right),
$$

where the constant in the $O$ only depends on $X_{1}, \ldots, X_{r}$ (and not on $\omega$ ). To prove this, it is sufficient to do it with the observables $X_{i}=\Sigma_{l_{i}}$, because they generate the algebra $\mathscr{O}$. Then, one can use a technique of conditioning to split the problem in two parts (see [Śni06, Proposition 4.1]):

1. showing the estimate for disjoint cumulants $k_{n, \omega}^{(r), \bullet}$, which are defined by the same formula as (1), except that the product in $\mathscr{O}$ is now the disjoint product (ii) $\Sigma_{\mu} \bullet \Sigma_{\nu}=\Sigma_{\mu \sqcup \nu}$. This is easy, as one can now compute explicitly the joint moments of observables $\Sigma_{\mu}$ with respect to this simpler product.

(i) The image in $\mathscr{P}$ of the isomorphism of graded algebras underlying this identification is the subalgebra of $\mathfrak{S}_{\infty}$-invariants for the action $\tau \cdot(\sigma, S)=\left(\tau \sigma \tau^{-1}, \tau(S)\right)$.

(ii) For two partitions $\mu$ and $\nu$, we denote in the following $\mu \sqcup \nu$ the partition whose parts are those of $\mu$ and those of $\nu$. 
2. compute the degree of the identity cumulants of observables, which are the observables defined by

$$
w^{(r)}\left(X_{1}, \ldots, X_{r}\right)=\sum_{\llbracket 1, r \rrbracket=\pi_{1} \sqcup \cdots \sqcup \pi_{s} \text { set partition }}(-1)^{s-1}(s-1) ! \prod_{\pi_{j} \in \pi}^{\bullet}\left[\prod_{i \in \pi_{j}} X_{i}\right],
$$

to be compared to Formula (1). This degree is of course smaller than $\operatorname{deg} X_{1}+\cdots+\operatorname{deg} X_{r}$, and by expanding the observables over the basis of symbols $\left(\Sigma_{\mu}\right)_{\mu \in \mathfrak{P}}$ and using the product rule (1), one can show that it is in fact $\operatorname{deg} X_{1}+\cdots+\operatorname{deg} X_{r}-(r-1)$. Indeed, a product of observables $\Sigma_{\mu^{(1)}} \cdots \Sigma_{\mu^{(r)}}$ is a sum over all partial matchings of the sets of indices $I\left(\mu_{1}\right), \ldots, I\left(\mu_{r}\right)$, whereas the cumulant $w^{(r)}\left(\Sigma_{\mu^{(1)}}, \ldots, \Sigma_{\mu^{(r)}}\right)$ is a sum restricted to "connected" matchings. This explains combinatorially the lost of a factor $n^{-(r-1)}$ in the estimate (2).

The estimate for cumulants follows then from the relation

$$
k_{n, \omega}^{(r)}\left(X_{1}, \ldots, X_{r}\right)=\sum_{\llbracket 1, r \rrbracket=\pi_{1} \sqcup \cdots \sqcup \pi_{s} \text { set partition }} k_{n, \omega}^{(s), \bullet}\left(w^{\left(\left|\pi_{1}\right|\right)}\left(X_{i \in \pi_{1}}\right), \ldots, w^{\left(\left|\pi_{s}\right|\right)}\left(X_{i \in \pi_{s}}\right)\right) .
$$

We refer to [Śni06, FM10] for more details on these techniques; notice that our result (2) is not the same as [Śni06, Theorem 3.1], because the gradation on $\mathscr{O}$ studied here is different from the one considered in Śniady's paper (actually, our case is much simpler). One obtains then that the $r$-th joint cumulant of variables $X_{n, \omega}(\sigma)$ is always a $O\left(n^{1-r / 2}\right)$, thus goes to zero when $r \geq 3$. When $r=2$, the computation of the limiting covariances amounts to understanding what are the two leading terms of a product $\Sigma_{\mu} \Sigma_{\nu}$ with respect to the gradation $\operatorname{deg} \Sigma_{\mu}=|\mu|$ on $\mathscr{O}$. This is a simple application of the product rule (1):

$$
\left.\Sigma_{\mu} \Sigma_{\nu}=\Sigma_{\mu \sqcup \nu}+\sum_{a \in \mu, b \in \nu} a b \Sigma_{\mu \sqcup \nu \sqcup(a+b-1) \backslash\{a, b\}}+\text { (terms of degree lower than }|\mu|+|\nu|-2\right) .
$$

This leads to the following algebraic central limit theorem, which can be seen as the analogue of Kerov's central limit theorem $(c f .[\mathrm{IO} 02, \S 6])$ in the case of central measures:

Theorem 1 For any parameter $\omega \in \Omega$, the random process $\left(X_{n, \omega}(\sigma)\right)_{\sigma \in \mathfrak{S}_{\infty}}$ converges in finite dimensional laws to a centered gaussian process $\left(X_{\infty, \omega}(\sigma)\right)_{\sigma \in \mathfrak{S}_{\infty}}$. If $\sigma$ and $\tau$ have cycle types $\mu$ and $\nu$, then

$$
\operatorname{cov}\left(X_{\infty, \omega}(\sigma), X_{\infty, \omega}(\tau)\right)=\sum_{a \in \mu, b \in \nu} a b p_{\mu \sqcup \nu \backslash\{a, b\}}(\omega)\left(p_{a+b-1}(\omega)-p_{a}(\omega) p_{b}(\omega)\right) .
$$

In particular, for two cycles of length $k$ and $l, \operatorname{cov}\left(X_{\infty, \omega}\left(c_{k}\right), X_{\infty}\left(c_{l}\right)\right)=k l\left(p_{k+l-1}(\omega)-p_{k}(\omega) p_{l}(\omega)\right)$.

Notice that the covariances are non-zero, except when $\omega=(0,0)$. In this case corresponding to Plancherel measures, Theorem 1 is not sufficiently precise, and the renormalization factor $n^{1 / 2}$ have in fact to be replaced by $n^{k / 2}$ for the study of the fluctuations of $\chi^{\lambda}\left(c_{k}\right)$; see again [I002].

Now, Theorem 1 gives a lot of information on the geometry of the random partitions $\lambda$. Indeed, the algebra of observables of Young diagrams linearly generated by the symbols $\Sigma_{\mu}$, also contains the functions

$$
p_{k}(\lambda)=\sum_{i=1}^{d}\left(\lambda_{i}-i+1 / 2\right)^{k}+(-1)^{k-1}\left(\lambda_{i}^{\prime}-i+1 / 2\right)^{k}=\sum_{i=1}^{d} a_{i}(\lambda)^{k}+(-1)^{k-1} b_{i}(\lambda)^{k},
$$


where $d$ is the size of the diagonal of the Young diagram of $\lambda$ (see [IO02, §1-4]; the $a_{i}(\lambda)$ 's and the $b_{i}(\lambda)$ 's are called the modified Frobenius coordinates of $\lambda$ ). Moreover, $p_{k}-\Sigma_{k}$ is always an observable of degree smaller than $k-1$; for example, $p_{4}-\Sigma_{4}=4 \Sigma_{2,1}+\frac{5}{2} \Sigma_{2}$. As a consequence, Theorem 1 holds with $X_{\infty, \omega}\left(c_{k}\right)$ replaced by $\sqrt{n}\left(n^{-k} p_{k}(\lambda)-p_{k}(\omega)\right)$, and it follows that the random discrete probability measure

$$
X_{\lambda}=\sum_{i=1}^{d}\left(a_{i}(\lambda) / n\right) \delta_{\left(a_{i}(\lambda) / n\right)}+\left(b_{i}(\lambda) / n\right) \delta_{\left(-b_{i}(\lambda) / n\right)}
$$

is asymptotically gaussian, in the following sense. Denote $X_{\omega}=\sum_{i=1}^{\infty} \alpha_{i} \delta_{\alpha_{i}}+\beta_{i} \delta_{-\beta_{i}}+\gamma \delta_{0}$. For any function $f \in \mathscr{C}^{1}([-1,1]), Y_{n, \omega}(f)=\sqrt{n}\left(X_{\lambda}(f)-X_{\omega}(f)\right)$ converges to a centered gaussian variable $Y_{\infty, \omega}(f)$, with covariances

$$
\operatorname{cov}\left(Y_{\infty, \omega}(f), Y_{\infty, \omega}(g)\right)=X_{\omega}\left((x f(x))^{\prime}(x g(x))^{\prime}\right)-X_{\omega}\left((x f(x))^{\prime}\right) X_{\omega}\left((x g(x))^{\prime}\right) .
$$

Choosing adequate test functions $f$ and $g$, when all the Dirac weights appearing in $X_{\omega}$ are distinct (i.e., $\alpha$ and $\beta$ are strictly decreasing), this leads to the following geometric counterpart of Theorem 1 ;

Theorem 2 Suppose $\alpha$ and $\beta$ decreasing. Then, the random variables $\Delta_{n, i}=\sqrt{n}\left(\lambda_{i} / n-\alpha_{i}\right)$ and $\Delta_{n, j}^{\prime}=\sqrt{n}\left(\lambda_{j} / n-\beta_{j}\right)$ converge jointly to a gaussian process $\left(\Delta_{\infty, i}, \Delta_{\infty, j}^{\prime}\right)_{i, j \geq 1}$, with covariances

$$
\begin{aligned}
& \operatorname{cov}\left(\Delta_{\infty, i}, \Delta_{\infty, k}\right)=\delta_{i k} \alpha_{i}-\alpha_{i} \alpha_{k} \quad ; \quad \operatorname{cov}\left(\Delta_{\infty, i}, \Delta_{\infty, j}^{\prime}\right)=-\alpha_{i} \beta_{j} \\
& \operatorname{cov}\left(\Delta_{\infty, j}^{\prime}, \Delta_{\infty, l}^{\prime}\right)=\delta_{j l} \beta_{j}-\beta_{j} \beta_{l} .
\end{aligned}
$$

Actually, it is clear from the proof that Theorem 2 remains true if one only considers the fluctuations of rows and columns corresponding to isolated parameters $\alpha_{i}$ and $\beta_{j}$, meaning that $\alpha_{i-1}>\alpha_{i}>\alpha_{i+1}$ or that $\beta_{j-1}>\beta_{j}>\beta_{j+1}$. The remaining of the paper is devoted to get rid of these hypotheses.

\section{From central measures to generalized riffle shuffles}

A point $\omega \in \Omega$ being fixed, consider a deck of cards ordered from 1 to $n$, and the following random algorithm:

1. First, we split the deck in ordered blocks of sizes $d_{1}+d_{2}+\cdots+e_{1}+e_{2}+\cdots+f=n$, the sizes of the blocks being chosen randomly according to the multinomial law of parameter $(\alpha, \beta, \gamma)$ :

$$
\mathbb{P}\left[d_{1}, d_{2}, \ldots, e_{1}, e_{2}, \ldots, f\right]=\left(\begin{array}{c}
n \\
d_{1}, d_{2}, \ldots, e_{1}, e_{2}, \ldots, f
\end{array}\right)\left(\prod_{i \geq 1} \alpha_{i}^{d_{i}}\right)\left(\prod_{j \geq 1} \beta_{j}^{e_{j}}\right) \gamma^{f} .
$$

2. In the blocks of size $e_{1}, e_{2}, \ldots$, we reverse the order of the cards. In the block of size $l$, the order of the cards is randomized, so that every permutation of the $l$ last cards becomes equiprobable.

3. Finally, we randomly shuffle the blocks back together, so that every shuffle that conserves the order of each block is equiprobable with probability $1 /\left(d_{d_{1}, d_{2}, \ldots, e_{1}, e_{2}, \ldots, f}\right)$. 
We denote by $\mathbb{Q}_{n, \omega}$ the probability measure on $\mathfrak{S}_{n}$ that corresponds to this random algorithm, called generalized riffle shuffle of parameter $\omega$. On the other hand, recall that the RSK algorithm yields a bijection between permutations $\sigma \in \mathfrak{S}_{n}$, and pairs $(P(\sigma), Q(\sigma))$ of standard tableaux of same shape $\Lambda(\sigma) \in \mathfrak{P}_{n}$. The underlying partition $\Lambda=\Lambda(\sigma)$ is related to the Greene invariants of $\sigma$ :

$$
\Lambda_{1}+\Lambda_{2}+\cdots+\Lambda_{r}=\max \left\{\ell\left(w_{1}\right)+\ell\left(w_{2}\right)+\cdots+\ell\left(w_{r}\right)\right\} \quad \text { for } r \geq 1
$$

where the maximum is taken over $r$-tuples $\left(w_{1}, \ldots, w_{r}\right)$ of disjoint increasing subwords of $\sigma$.

Theorem 3 The image of the probability measure $\mathbb{Q}_{n, \omega}$ by the $R S K$ map $\Lambda: \mathfrak{S}_{n} \rightarrow \mathfrak{P}_{n}$ is $\mathbb{P}_{n, \omega}$.

This result appears in [Ful02, Theorem 13], and it can be given an elegant proof by using the combinatorial Hopf algebra FQSym, also known as the Malvenuto-Reutenauer algebra (see [DHT01, §3.6], which states the simpler case when $\beta=\gamma=0$ ). This Hopf algebra is linearly generated by elements $F_{\sigma}$ labelled by all the permutations in $\mathfrak{S}=\bigsqcup_{n \in \mathbb{N}} \mathfrak{S}_{n}$, with the following product rule:

$$
F_{\sigma} F_{\tau}=\sum_{v \in \sigma \bar{\varpi} \tau} F_{v},
$$

where $\sigma \bar{\amalg} \tau$ denotes the product of shifted shuffle of $\sigma \in \mathfrak{S}_{n}$ and $\tau \in \mathfrak{S}_{p}$, that is to say the set of permutations that are obtained by shuffling the two words $\sigma(1) \cdots \sigma(n)$ and $(n+\tau(1)) \cdots(n+\tau(p))$. The scalar product $\left\langle F_{\sigma} \mid F_{\tau}\right\rangle=\mathbf{1}_{|\sigma|=|\tau|} \mathbf{1}_{\sigma \tau^{-1}=\text { id }}$ endows FQSym with a structure of noncommutative selfdual graded Hopf algebra, whose coproduct can be written in terms of the operations of deconcatenation and standardization (see e.g. [DHT01, §2.1] for details on the operation of standardization of words):

$$
\Delta\left(F_{\sigma}\right)=\sum_{\sigma=\tau \cdot \nu} F_{\operatorname{Std}(\tau)} \otimes F_{\operatorname{Std}(\nu)}
$$

For instance, $\Delta\left(F_{4132}\right)=1 \otimes F_{4132}+F_{1} \otimes F_{132}+F_{21} \otimes F_{21}+F_{312} \otimes F_{1}+F_{4132} \otimes 1$. Now, an important result is that FQSym admits a realization as an algebra of formal power series in noncommutative variables. If $X=\left\{x_{1}, x_{2}, \ldots\right\}$ is an alphabet of noncommutative variables, we view each monomial $x_{i_{1}} x_{i_{2}} \cdots x_{i_{r}}$ as a word $w=i_{1} i_{2} \ldots i_{r}$. Then, the identification

$$
F_{\sigma^{-1}}=G_{\sigma}=\sum_{w \mid \operatorname{Std}(w)=\sigma} w
$$

provides a realization of FQSym, see [DHT01]. As a consequence, to any sequence $\alpha=\left(\alpha_{i}\right)_{i \geq 1}$ of complex numbers whose series is absolutely convergent, one can associate the specialization of FQSym given by $F_{\sigma}(\alpha)=F_{\sigma}\left(\alpha_{1}, \alpha_{2}, \ldots\right)$ - one replaces each variable $x_{i}$ by $\alpha_{i}$. On the other hand, other specializations FQSym $\rightarrow \mathbb{C}$ are given by the so-called "exponential alphabet" and its multiples:

$$
F_{\sigma}(\gamma E)=\frac{\gamma^{|\sigma|}}{|\sigma| !}, \quad \text { where }|\sigma|=n \text { if } \sigma \in \mathfrak{S}_{n} .
$$

By using the Hopf algebra structure, one can then associate to any $\omega \in \Omega$ a specialization $F \mapsto F(\omega)$ of FQSym:

$$
F(\omega)=[(\mathrm{id} \otimes S \otimes \mathrm{id}) \circ(\Delta \otimes \mathrm{id}) \circ \Delta] F(\alpha,-\beta, \gamma E),
$$


where $S$ is the antipode of FQSym, and $(\alpha,-\beta, \gamma E)$ is the tensor product of the aforementioned specializations, and therefore a morphism of algebras from $\mathbf{F Q S y m}^{\otimes 3}$ to $\mathbb{C}$. These specializations have the two following important properties:

(i) Denote $\pi$ the morphism from FQSym to $\mathbb{C}[[X]]$ that makes the variables $x_{1}, x_{2}, \ldots$ commutative. The image of FQSym by $\pi$ is the algebra of quasisymmetric functions QSym, and for any partition $\lambda \in \mathfrak{P}_{n}$,

$$
(\operatorname{dim} \lambda) s_{\lambda}=\sum_{\sigma \in \mathfrak{S}_{n} \mid \Lambda(\sigma)=\lambda} \pi\left(F_{\sigma}\right)=\sum_{\sigma \in \mathfrak{S}_{n} \mid \Lambda(\sigma)=\lambda} \pi\left(G_{\sigma}\right) .
$$

As a consequence, since $\pi$ commutes with the specializations associated to the points $\omega \in \Omega$,

$$
\mathbb{P}_{n, \omega}[\lambda]=(\operatorname{dim} \lambda) s_{\lambda}(\omega)=\sum_{\sigma \in \mathfrak{S}_{n} \mid \Lambda(\sigma)=\lambda} G_{\sigma}(\omega) .
$$

(ii) For any $\sigma \in \mathfrak{S}_{n}, G_{\sigma}(\omega)=\mathbb{Q}_{n, \omega}(\sigma)$. This can be proved by interpreting the three steps of the random algorithm as linear operations between FQSym and tensor products of it - one identifies a probability measure $\mathbb{P}$ on permutations as the element $\sum_{\sigma \in \mathfrak{S}_{n}} \mathbb{P}[\sigma] F_{\sigma}$ of FQSym. More precisely:

1. The operation of cutting the deck in random blocks is related to the coproduct of FQSym and to the specializations $\alpha$ and $-\beta$.

2. The randomization of the last block is related to the specialization $\gamma E$, and the reversal of some blocks is related to the antipode of FQSym - indeed, $S\left(F_{12 \cdots n}\right)= \pm F_{n \cdots 21}$.

3. The shuffle of the blocks is obviously related to the multiplication of the algebra.

These two points proves immediately Theorem 3 , and they also give an abstract formula for $\mathbb{Q}_{n, \omega}(\sigma)$ as a specialization of a certain element in FQSym, or of its commutative version QSym. Notice that Theorem 3 gives a combinatorial motivation for the study of the models of random partitions $\left(\mathbb{P}_{n, \omega}\right)_{n \in \mathbb{N}}$ : indeed, Formula (3) shows that these models encode the structure of increasing subwords in shuffles.

\section{Fluctuations of the rows and columns of the random partitions}

The combinatorial interpretation of the central measures shown in the previous paragraph yields a second "geometric" central limit theorem, which solves the case of equal parameters at the expense of the hypothesis (iii) $\beta=\gamma=0$. To state this result, it is convenient to rewrite the sequence $\left(\alpha_{i}\right)_{i \geq 1}$ as $\left(p_{1}^{m_{1}}>p_{2}^{m_{2}}>\cdots\right)$, where each parameter $p_{i}$ appears with multiplicity $m_{i}$, so $p_{1}=\alpha_{1}=\cdots=\alpha_{m_{1}}$, $p_{2}=\alpha_{m_{1}+1}=\cdots=\alpha_{m_{1}+m_{2}}$, etc. We reindex similarly the rows of $\lambda$, and write the parts

$$
\lambda=\left(\lambda_{1,1} \geq \lambda_{1,2} \geq \cdots \geq \lambda_{1, m_{1}} \geq \lambda_{2,1} \geq \cdots \geq \lambda_{2, m_{2}} \geq \cdots\right) .
$$

Theorem 4 We assume $\beta=\gamma=0$, and we denote $\Delta_{n, i, j}=\sqrt{n}\left(\lambda_{i, j} / n-p_{i}\right)$ the fluctuations of the $m=m_{1}+\cdots+m_{r}$ first rows. These fluctuations converge in joint laws towards the random variables

$$
\Delta_{\infty, i, j}=\sqrt{p_{i}}\left(X_{i}+Y_{i, j}\right)
$$

(iii) Notice however that one can rigourously adapt Theorem 4 to the case when $\beta$ has a finite number of non-zero parameters. 
where $\left(X_{1}, \ldots, X_{r}\right)$ is a centered gaussian vector of covariance matrix $\left(\delta_{i k} / m_{i}-\sqrt{p_{i} p_{k}}\right)_{1 \leq i, k \leq r}$, and for each $i \in \llbracket 1, r \rrbracket$,

$$
\left(Y_{i, 1}, \ldots, Y_{i, m_{i}}\right) \sim \mathbf{1}_{\begin{array}{r}
y_{1} \geq y_{2} \geq \cdots \geq y_{m_{i}} \\
y_{1}+y_{2}+\cdots+y_{m_{i}}=0
\end{array}} \frac{\prod_{1 \leq j<l \leq m_{i}}\left(y_{j}-y_{l}\right)^{2}}{1 ! 2 ! \cdots\left(m_{i}-1\right) !(2 \pi)^{\frac{m_{i}-1}{2}}} \mathrm{e}^{-\frac{\|y\|^{2}}{2}} d^{m_{i}-1} y
$$

and is independent of the gaussian vector and of the other vectors $\left(Y_{k, 1}, \ldots, Y_{k, m_{k}}\right)$. In other words, each vector $Y_{i}$ is distributed as the eigenvalues of a gaussian traceless Hermitian matrix $M_{i}$ of size $m_{i} \times m_{i}$ and covariance matrix $\mathbb{E}\left[\operatorname{tr}\left(\left(A M_{i}\right)\left(B M_{i}\right)^{\dagger}\right)\right]=\left(\left(m_{i}^{2}-1\right) / m_{i}\right) \operatorname{tr}\left(A B^{\dagger}\right)$.

Let us sketch the proof of this result; we shall even assume that $\omega=(\alpha, 0)$ has only a finite number $m$ of non-zero parameters, but this is only in order to simplify the notations, and not really required by the proof. To begin with, notice that the shape $\Lambda(\sigma)$ of the partition associated to a shuffle $\sigma$ of blocks of size $d_{1}, \ldots, d_{m}$ only depends on the original blocks of each letter $\sigma(1), \sigma(2)$, etc., and not on the precise values $\sigma(1), \sigma(2)$, etc. Indeed, the permutation $\sigma$ is equal to the standardized of the word that encodes the original blocks, and the standardization does not change the shape of the tableaux. Consider then independent random variables $w_{1}, w_{2}, \ldots$ such that $\mathbb{P}\left[w_{j}=i\right]=\alpha_{i}$ for any indices $i, j \geq 1$. The previous discussion and Theorem 3 show that a way to realize a random partition $\lambda \sim \mathbb{P}_{n, \omega}$ is to apply the $\operatorname{RSK}$ map $\Lambda$ to the word $w_{1} w_{2} \ldots w_{n}$. We then associate to any random word $w=w_{1} w_{2} \ldots$ a random walk $\left(X^{(n)}\right)_{n \in \mathbb{N}}$ in $\mathbb{Z}^{m}$ by setting

$$
X^{(0)}=(0, \ldots, 0) \quad ; \quad X^{(n)}-X^{(n-1)}=e_{i}=\left(0^{1}, \ldots, 0^{i-1}, 1^{i}, 0^{i+1}, \ldots, 0^{m}\right)
$$

if $w_{n}=i$. On the other hand, we denote by $\lambda^{(n)}=\left(\lambda_{1}^{(n)}, \ldots, \lambda_{m}^{(n)}\right)$ the shape of the tableaux obtained from $w_{1} \ldots w_{n}$ by the RSK algorithm (possibly with the last parts equal to 0 ). These two discrete random paths interpolate continuous piecewise affine paths $\left(X^{(t)}\right)_{t \in \mathbb{R}_{+}}$and $\left(\lambda^{(t)}\right)_{t \in \mathbb{R}_{+}}$in $\mathscr{C}^{0}\left(\mathbb{R}_{+}, \mathbb{R}^{m}\right)$.

A theorem due to O'Connell ([O'C03, BBO05]) explains how to go from $\left(X^{(t)}\right)_{t \in \mathbb{R}_{+}}$to $\left(\lambda^{(t)}\right)_{t \in \mathbb{R}_{+}}$by using Pitman transforms. To each simple root $\delta_{i}=e_{i}-e_{i+1}$ of the root system of type $A_{m-1}$, we attach an operator $P_{i}: \mathscr{C}^{0}\left(\mathbb{R}_{+}, \mathbb{R}^{m}\right) \rightarrow \mathscr{C}^{0}\left(\mathbb{R}_{+}, \mathbb{R}^{m}\right)$ defined by

$$
\left(P_{i} \gamma\right)(t)=\gamma(t)-\left(\min \left\{\left\langle\delta_{i} \mid \gamma(s)\right\rangle \mid s \leq t\right\}\right) \delta_{i}
$$

These operators satisfy the braid relations of $\mathfrak{S}_{m}$; as a consequence, if a permutation $\sigma \in \mathfrak{S}_{m}$ has a decomposition of minimal length $\sigma=s_{i_{1}} s_{i_{2}} \cdots s_{i_{l}}$ with $s_{i}=(i, i+1)$, then $P_{\sigma}=P_{i_{1}} P_{i_{2}} \cdots P_{i_{l}}$ does not depend on the decomposition and is thus well defined. In particular, one can consider $G_{m}=P_{\omega_{0}}$ with $\omega_{0}=m(m-1) \ldots 21$, and this operator has the following fundamental properties:

P1 It is idempotent with values in $\mathscr{C}^{0}\left(\mathbb{R}_{+}, C\right)$, where $C=\left\{x \mid x_{1} \geq x_{2} \geq \cdots \geq x_{m}\right\}$. Also, it stabilizes $\mathscr{C}^{0}\left(\mathbb{R}_{+}, H\right)$, where $H=\left\{x \mid x_{1}+x_{2}+\cdots+x_{m}=0\right\}-$ this is true for any $P_{i}$.

P2 It transforms a standard $(m-1)$-dimensional Brownian motion on $H$ into a Brownian motion conditioned in Doob's sense to stay in the Weyl chamber $C \cap H$, see [BBO05, Theorem 5.6]. The densities of such a conditioned Brownian motion are given (up to a scalar) by the formula of Theorem 4

P3 The effect of the RSK map is given by the relation $\lambda^{(t)}=\left(G_{m} X\right)^{(t)}, c f$. [O'C03, Theorem 3.1]. 
Example. We have drawn below:

1. the recentered random walk $X^{(t)}-t \frac{e_{1}+e_{2}+e_{3}}{3}$ associated to a random word of parameters $\alpha_{1}=$ $\alpha_{2}=\alpha_{3}=1 / 3$

2. its image by Pitman's transform $G_{3}$, which is also $\lambda^{(t)}-t \frac{e_{1}+e_{2}+e_{3}}{3}$ (both curves stay in the hyperplane $H=\left\{x_{1}+x_{2}+x_{3}=0\right\}$, the second curve stay in a Weyl chamber).

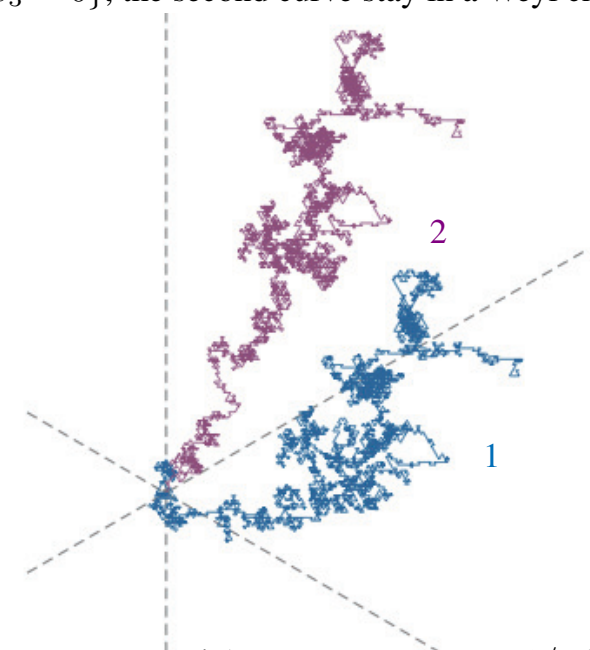

In the case when all the parameters are equal $\left(\alpha_{1}=\cdots=\bar{\alpha}_{m}=1 / m\right), \mathrm{P} 2$ and $\mathrm{P} 3$ imply readily our theorem, after some transformations of $X^{(t)}$ and $\lambda^{(t)}$ and by applying Donsker's theorem that states that the random process based on the rescaled means of i.i.d. random variables converge to a Brownian motion. When different parameters $p_{1}>\cdots>p_{r}$ appear with multiplicities $m_{1}+\cdots+m_{r}=m$, one has first to show the following technical lemma:

$$
G_{m}\left(X_{1}, \ldots, X_{m}\right)-\left(G_{m_{1}}\left(X_{1}, \ldots, X_{m_{1}}\right), \ldots, G_{m_{r}}\left(X_{m_{1}+\cdots+m_{r-1}+1}, \ldots, X_{m}\right)\right)
$$

have all its coordinates that remain almost surely bounded. This is mainly because in each block of coordinates of size $m_{i}$, the $X_{j}$ 's have a different rate of growth $p_{i}$. As a consequence, each Pitman operator $P_{k}$ with $k=m_{1}+\cdots+m_{j \in \llbracket 1, r-1 \rrbracket}$ modifies the random process $\left(X^{(t)}\right)_{t \in \mathbb{R}^{d}}$ only by an almost surely bounded process. This allows to simplify the operator $G_{m}$ and split it (up to a bounded process) in $G_{m_{1}} \otimes G_{m_{2}} \otimes \cdots \otimes G_{m_{r}}$. Then, one can use the same techniques as in the case when all the $\alpha_{i}$ 's are equal, which proves Theorem 4

\section{Perspectives (mod-gaussian convergence)}

To conclude this paper, let us evoke a possible improvement of the techniques detailed in Section 1 . that relies on the estimate (2) and on the notion of mod-gaussian convergence ([JKN11, DKN11]). A sequence of centered random variables $\left(Z_{n}\right)_{n \in \mathbb{N}}$ is said to converge in the mod-gaussian sense with parameters $\left(t_{n}\right)_{n \in \mathbb{N}}$ and $\Phi$ if for all $u \in \mathbb{R}$,

$$
\mathrm{e}^{\frac{t_{n} u^{2}}{2}} \mathbb{E}\left[\mathrm{e}^{\mathrm{i} u Z_{n}}\right] \rightarrow \Phi(u)
$$


where $\Phi$ is a continuous function with $\Phi(0)=1$, and the convergence in 4 holds locally uniformly in $u$. A typical case is when $Z_{n}=X_{n}+Y_{n}$ with $X_{n}$ gaussian of variance $t_{n}$, and $Y_{n}$ independent of $X_{n}$ and that converges in law towards a random variable of characteristic function $\Phi$; however, this situation is not at all generic. Notice that one allows the "variances" $\left(t_{n}\right)_{n \in \mathbb{N}}$ to go to infinity; the idea being of rescaling differently the random variables under study and to go beyond the usual central limit theorem. We refer to [JKN11] for various examples of this new type of convergence of random variables (including examples from number theory and combinatorics), and to [DKN11] for a generalization of the notion and for the probabilistic consequences of the hypothesis of mod-gaussian convergence. In a few words, when mod-gaussian convergence is satisfied with $\left(t_{n}\right)_{n \in \mathbb{N}}$ growing to infinity, one gets:

MG1 a central limit theorem: $\left(\frac{Z_{n}}{\sqrt{t_{n}}}\right)_{n \in \mathbb{N}}$ converges to a centered gaussian variable of variance 1 .

MG2 a local limit theorem: for any real numbers $a<b, \mathbb{P}\left[a \leq Z_{n} \leq b\right]$ is equivalent to $\frac{b-a}{\sqrt{2 \pi t_{n}}}$ (this is not a direct consequence of the central limit theorem).

MG3 a principle of large deviations. We assume that the characteristic functions $\mathbb{E}\left[\mathrm{e}^{z Z_{n}}\right]$ are well defined on a band $B_{c, d}=\{z \mid-c<\operatorname{Re} z<d\}$, and not only on the line $z=\mathrm{i} u$ with $u \in \mathbb{R}$. We also assume that for $z \in B_{c, d}$,

$$
\mathrm{e}^{-\frac{t_{n} z^{2}}{2}} \mathbb{E}\left[\mathrm{e}^{z Z_{n}}\right] \rightarrow \Psi(z)
$$

with $\Psi$ analytic on the band and the convergence that is locally uniform in $z$ (strong mod-gaussian convergence). Then, one has the following (precise) principle of large deviations:

$$
\left.\mathbb{P}\left[\frac{Z_{n}}{\sqrt{t_{n}}} \geq \sqrt{t_{n}} x\right] \simeq \frac{\Psi(x)}{\sqrt{2 \pi t_{n} x^{2}}} \mathrm{e}^{-\frac{t_{n} x^{2}}{2}} \quad \text { for } x \in\right] 0, d[.
$$

We refer $e . g$. to [DKN11] for proofs of these facts. Now, let us see why this notion is interesting in the setting of central measures. In Section 1, we have only used the asymptotic vanishing of the cumulants $k^{(r)}$ of order $r \geq 3$ to prove the asymptotic gaussian behaviour of the variables $n^{1 / 2}\left(\chi^{\lambda}(\sigma)-\chi^{\omega}(\sigma)\right)$; but we have not used the precise estimate (2), and in particular the fact that the leading power of $n$ of the $r$-th cumulant of a scaled observable $X / n^{\mathrm{deg} X}$ is strictly decreasing in $r$. A careful use of this fact leads to the following conjecture. Fix an integer $k \geq 2$, and consider the variable $Z_{n, k}=n^{2 / 3}\left(\chi^{\lambda}\left(c_{k}\right)-\chi^{\omega}\left(c_{k}\right)\right)$. Then, its $r$-th cumulant is of order $O\left(n^{1-r} / 3\right)$, so, if we had a good control (in $r \geq 4$ ) of the constant involved in the $O$, we could prove the locally uniform convergence

$$
\log \mathbb{E}_{n, \omega}\left[\mathrm{e}^{z Z_{n, k}}\right]-n^{1 / 3} c_{2, k}(\omega) z^{2} \rightarrow c_{3, k}(\omega) z^{3}
$$

where $2 c_{2, k}=k^{2}\left(p_{2 k-1}-p_{k, k}\right)$ and $6 c_{3, k}=k^{3}\left((3 k-2) p_{3 k-2}-(6 k-3) p_{2 k-1, k}+(3 k-1) p_{k, k, k}\right)$ are the leading coefficients of the cumulants $k_{n, \omega}^{(2)}\left(\Sigma_{k}, \Sigma_{k}\right)$ and $k_{n, \omega}^{(3)}\left(\Sigma_{k}, \Sigma_{k}, \Sigma_{k}\right)$. Thus, precise bounds of the constants involved in (2) would imply the following conjecture:

Conjecture 5 For any $\omega \in \Omega$, the rescaled character values $Z_{n, k}$ with $\lambda \sim \mathbb{P}_{n, \omega}$ converge in the (strong) mod-gaussian sense with parameters $t_{n} \propto n^{1 / 3}$ and $\Psi(x)=\exp \left(c_{3, k}(\omega) x^{3}\right)$.

Then, by using MC2 and MG3, one would get new estimates on the distribution of the random characters, and possibly also on the distribution of the rows and columns. Notice that an analoguous conjecture can be made in the special case $\omega=(0,0)$ (Plancherel measures), but this time with $Z_{n, k}=n^{k / 2+1 / 6} \chi^{\lambda}\left(c_{k}\right)$ when $k$ is odd and $Z_{n, k}=n^{k / 2+1 / 4} \chi^{\lambda}\left(c_{k}\right)$ when $k$ is even. 


\section{Acknowledgements}

The author would like to express his gratitude to P. Biane, V. Féray, S. Giraudo, F. Hivert, A. Nikeghbali, P. Śniady, J.-Y. Thibon and A. Vershik for various discussions, comments and suggestions.

\section{References}

[BBO05] P. Biane, P. Bougerol, and N. O'Connell. Littelmann paths and Brownian paths. Duke Math. J., 130(1):127-167, 2005.

[Buf11] A. Bufetov. The central limit theorem for extremal characters of the infinite symmetric group. arXiv:1105.1519v2 [math.RT], 2011.

[DHT01] G. Duchamp, F. Hivert, and J.-Y. Thibon. Noncommutative symmetric functions VI: Free quasi-symmetric functions and related algebras. arXiv:math/0105065v1, 2001.

[DKN11] F. Delbaen, E. Kowalski, and A. Nikeghbali. Mod- $\varphi$ convergence. arXiv:1107.5667v2 [math.PR], 2011.

[FM10] V. Féray and P.-L. Méliot. Asymptotics of $q$-Plancherel measures. To appear in Probability Theory and Related Fields, arXiv:1001.2180v1 [math.RT], 2010.

[Ful02] J. Fulman. Applications of symmetric functions to cycle and increasing subsequence structure after shuffles. J. Alg. Combin., 16:165-194, 2002.

[IK99] V. Ivanov and S. Kerov. The algebra of conjugacy classes in symmetric groups, and partial permutations. In Representation Theory, Dynamical Systems, Combinatorial and Algorithmical Methods III, volume 256 of Zapiski Nauchnyh Seminarov POMI, pages 95-120, 1999.

[IO02] V. Ivanov and G. Olshanski. Kerov's central limit theorem for the Plancherel measure on Young diagrams. In Symmetric Functions 2001, volume 74 of NATO Science Series II. Mathematics, Physics and Chemistry, pages 93-151, 2002.

[JKN11] J. Jacod, E. Kowalski, and A. Nikeghbali. Mod-Gaussian convergence: new limit theorems in probability and number theory. Forum Math., 23(4):835-873, 2011.

[KV81] S. V. Kerov and A. M. Vershik. Asymptotic theory of characters of the symmetric group. Funct. Anal. Appl., 15(4):246-255, 1981.

[Mél11] P.-L. Méliot. A central limit theorem for the characters of the infinite symmetric group and of the infinite Hecke algebra. arXiv:1105.0091 [math.RT], 2011.

[O'C03] N. O'Connell. A path-transformation for random walks and the Robinson-Schensted correspondence. Trans. Amer. Math. Soc., 355:3669-3697, 2003.

[Śni06] P. Śniady. Gaussian fluctuations of characters of symmetric groups and of Young diagrams. Probab. Th. Rel. Fields, 136(2):263-297, 2006.

[Tho64] E. Thoma. Die unzerlegbaren, positive-definiten Klassenfunktionen der abzählbar unendlichen symmetrischen Gruppe. Math. Zeitschrift, 85:40-61, 1964. 